Euskal ikerketen aldizkaria | Revue d'études basques |

Revista de estudios vascos | Basque studies review

$10 \mid 2005$

Numéro $X$

\title{
L'enseignement suffit-il à " sauver » une langue menacée ? L'exemple du Pays Basque
}

Jean-Baptiste Coyos

\section{OpenEdition}

\section{Journals}

Édition électronique

URL : http://journals.openedition.org/lapurdum/40

DOI : $10.4000 /$ lapurdum.40

ISSN : 1965-0655

Éditeur

IKER

Édition imprimée

Date de publication : 31 décembre 2005

Pagination : 19-34

ISBN : 2-86781-409-X

ISSN : 1273-3830

Référence électronique

Jean-Baptiste Coyos, «L'enseignement suffit-il à « sauver » une langue menacée ? L'exemple du Pays Basque », Lapurdum [En ligne], 10 | 2005, mis en ligne le 01 avril 2008, consulté le 19 septembre 2020. URL : http://journals.openedition.org/lapurdum/40 ; DOI : https://doi.org/10.4000/lapurdum.40 


\title{
L'ENSEIGNEMENT SUFFIT-IL À "SAUVER" UNE LANGUE MENACÉE ? L'EXEMPLE DU PAYS BASQUE
}

\author{
Jean-Baptiste COYOS \\ IKER UMR 5478 CNRS \\ jean-baptiste.coyos@wanadoo.fr
}

\section{Introduction : quel(s) objectif(s) donne-t-on à l'enseignement de la langue ?}

Cette contribution prend pour point de départ le texte introductif aux exposés du colloque, intitulé "Problématiques linguistiques et enseignement ${ }^{1}$. Je cite : "Quelle que soit la région et la problématique originale de sa "langue régionale" (...) les questionnements didactique et pédagogique convergent vers une même interrogation partagée et centrale, qui appelle une réponse tout à la fois pragmatique et de principe et qui sollicite les sociolinguistes et les collectivités territoriales tout autant que les pédagogues. (...) Car au-delà de ses enjeux étroitement scientifiques et éducatifs, la question soulevée est celle des objectifs mêmes de l'enseignement d'une langue régionale comme aussi celle de ses finalités, c'est-à-dire de sa visée culturelle, sociale, économique et politique ».

Voici donc quelques réflexions concernant un objectif qui est souvent attribué à l'enseignement des langues menacées, celui tout simplement de la sauver. On ne discutera pas le sens de sauver une langue. Après tout une langue n'est morte que quand son dernier locuteur a disparu. Considérons plutôt, et c'est le cas dans une plus ou moins grande mesure de toutes les langues régionales de France, comme langue en danger une langue qui n'est plus ou très peu transmise en famille. Dans ces situations, on compte souvent sur l'enseignement scolaire de la langue, et celui aux adultes, pour empêcher la substitution complète de cette langue par la langue dominante. Ce que Joshua Fishman a appelé l' « inversion du glissement linguistique » (Reversing Language Shift : 1991, 2001). Et parfois tous les efforts sont mis dans le développement de l'enseignement, sans se préoccuper de tous les autres domaines qui concourent au renforcement de la connaissance et de la pratique de la langue, le but ultime étant selon les spécialistes de rétablir un taux suffisant de transmission intergénérationnelle pour considérer qu'une langue n'est pas en danger.

La question posée peut être divisée en plusieurs et nous allons l'illustrer avec la situation au Pays Basque. L'enseignement d'une langue dominée, en danger, est-il un levier suffisant pour rétablir la transmission intergénérationnelle? La connaissance de la langue implique-t-il son usage ? Dit autrement, connaissance et usage vont-ils de pair ? Quelles sont les conditions pour que la langue apprise ne soit pas seulement une langue d'enseignement, une matière parmi d'autres comme les mathématiques ou la géographie ? La question est vaste et nous ne ferons que l'aborder.

\footnotetext{
Cet article fait suite à une communication du même nom faite le 27 octobre 2004 à Saverne (Alsace), lors du premier congrès professionnel intitulé « Un professorat de langue régionales pour le premier degré », organisé par l'association Lehrer (Association professionnelle des instituteurs et professeurs pour l'enseignement bilingue paritaire dans les Académies de Strasbourg et Nancy-Metz) et l'Office pour la langue et la culture d'Alsace.
}

1 
Voyons tout d'abord brièvement quelle est la situation de l'enseignement de la langue basque des deux côtés de la frontière, puis quel est usage qui est fait de la langue, dans la mesure où ceci est quantifiable, enfin quel est l'écart entre la connaissance et l'usage de la langue. Dans un dernier temps, nous esquisserons un début de réponse à la question posée en titre.

\section{L’enseignement de la langue basque : effectifs, évolution}

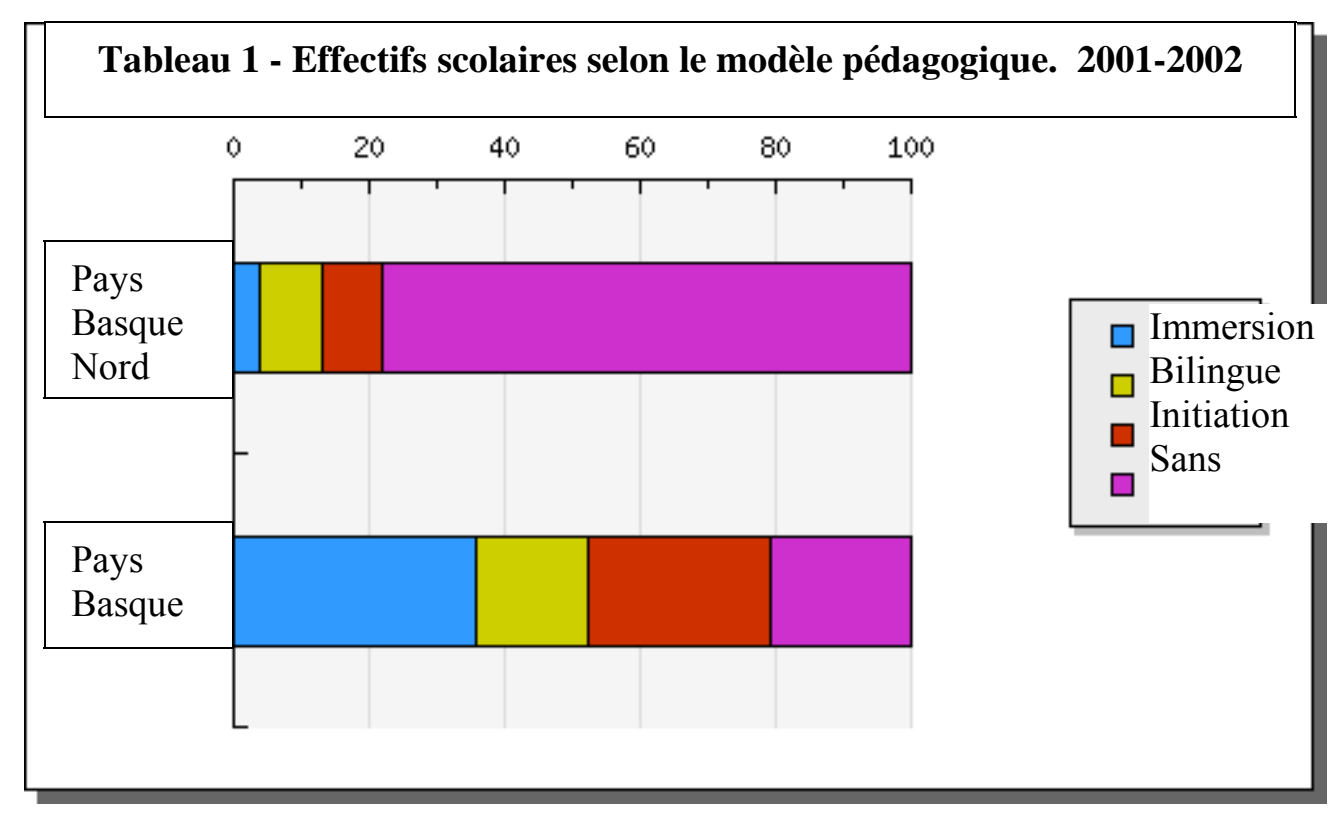

Source : Euskararen Datu-Basea, Euskal Soziolinguistika Institutua²

Comme on peut le voir dans le tableau 1 «Effectifs scolaires selon le modèle pédagogique ", pour l'ensemble du Pays Basque, Euskadi, Navarre et Pays Basque Nord (Pays Basque de France) pour l'année scolaire 2001-2002, la proportion d'élèves sans aucun enseignement de basque est d'environ $20 \%$. En Pays Basque Nord, elle atteint près de $70 \%{ }^{3}$.

Dès la fin de la période franquiste, de très gros efforts ont été fournis dans la Communauté autonome basque d'Euskadi (les provinces d'Alava / capitale Vitoria, de Biscaye / capitale Bilbao et du Guipúzcoa / capitale Saint-Sébastien), afin de développer l'enseignement de la langue. L'enseignement du basque est généralisé en Guipúzcoa, du moins en maternelle et primaire, sous une forme ou une autre, avec l'enseignement immersif majoritaire $(\approx 60 \%)$.

Pour l'année scolaire 2004-2005, en Pays Basque Nord, au contraire, l'enseignement immersif ne concerne que $7 \%$ des enfants scolarisés. On arrive à un taux de $24,7 \%$ d'enfants scolarisés avec le modèle immersif ou bilingue en maternelle et primaire en France, initiation non comprise donc. En collège les modèles immersif et bilingue concernent 9,9\% des élèves, en lycée général et technologique $4,7 \%$ des élèves et en lycée professionnel $0,3 \%$.

\footnotetext{
${ }^{2}$ Tous les tableaux sont issus de cette même source.

${ }^{3}$ On trouvera toutes les données concernant le Pays Basque de France pour l'année scolaire 2004-2005 dans l'étude de l'Office public da la langue basque, Diagnostic de l'offre d'enseignement... L'étude intitulée L'offre d'enseignement du basque et en basque... propose une étude secteur par secteur et une ébauche de programmation annuelle. Nous laissons de côté la Communauté autonome de Navarre qui se trouve dans une situation intermédiaire.
} 

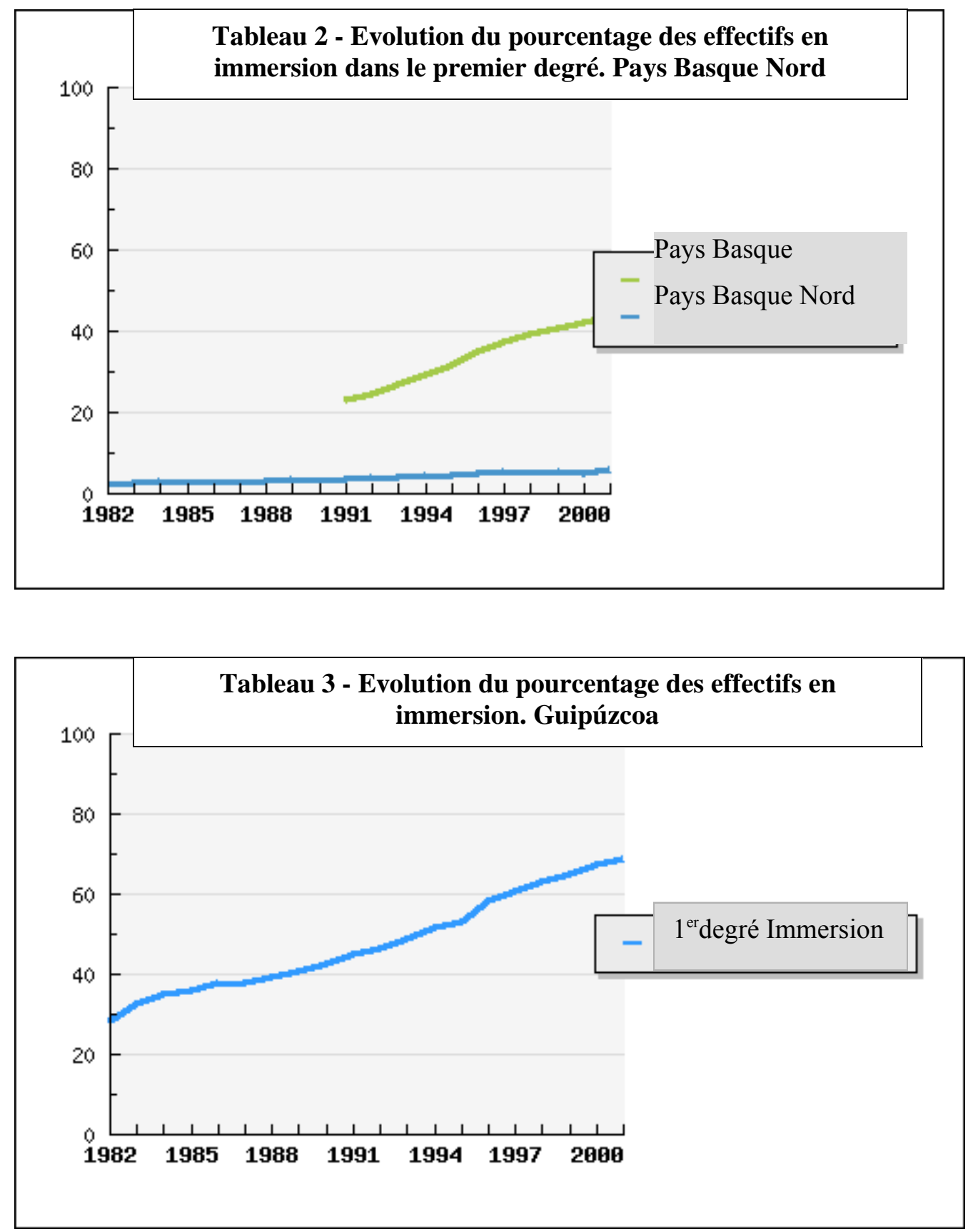

En comparant les tableaux 2 et 3 intitulés « Evolution du pourcentage des effectifs en immersion ", on constate que le modèle pédagogique immersif est en constante progression mais dans des proportions très différentes selon la zone géographique considérée, Pays Basque Nord, ensemble du Pays Basque, ou Guipúzcoa qui est la province où l'enseignement de la langue basque est le plus développé ${ }^{4}$. Pour l'ensemble d'Euskadi en 2005-2006 on atteint $52,3 \%$ dans le modèle immersif, contre $16,2 \%$ en $1985-1986$, avec $62,7 \%$ en maternelle (3-5 ans), 56,5\% en primaire, $48,4 \%$ en collège et $32,5 \%$ pour les $16-18$ ans s. $^{5}$.

C'est en Pays Basque Nord que l'enseignement par immersion progresse le moins rapidement (tableau 2 ci-dessous). En 2004-2005 il concerne 1518 enfants en maternelle et primaire soit $6,3 \%$ des élèves de maternelle et $5,4 \%$ des élèves du primaire ; en collège l'immersion représente $3,7 \%$ des élèves et en lycée $2,4 \%$.

\footnotetext{
${ }^{4}$ La courbe concernant le Pays Basque dans son ensemble ne démarre qu'en 1991.

${ }^{5}$ Données de Euskaraz nahi dugu hitz eta jolas, 15/09/2005, www.erabili.com/zer_berri/berriak/
} 


\section{3. Évaluation de la connaissance et de l'usage de la langue basque}

Si les effectifs scolaires selon les modèles d'enseignement sont parfaitement connus, il est par contre difficile, on le sait, d'évaluer la connaissance et l'usage de la langue. Des enquêtes sociolinguistiques régulières sont menées à l'initiative du Gouvernement autonome basque et de son vice-ministère à la Politique linguistique, tous les 5 ans, sur l'ensemble du Pays Basque depuis 1991. Ce sont de vastes enquêtes sur des bases déclaratives et donc, ceci est bien connu, même si l'enquêteur est bascophone et converse en basque avec l'enquêté, ce sont d'abord les opinions, les représentations des enquêtés que l'on recueille.

Mais malgré leurs limites, ces enquêtes restent tout de même de bons indicateurs qui permettent de dépasser l'empirisme qui entoure généralement la situation des langues dites de France, en dehors du français justement.

\section{1. Évaluation de la connaissance de la langue basque}

Le tableau 4 ci-dessous provient de la dernière enquête de 2001. On y remarque que le Pays Basque Nord compte $24,7 \%$ de bilingues et $9,3 \%$ de bilingues dits passifs qui sont des personnes qui comprennent plus ou moins le basque mais qui ne le parlent pas. Les monolingues francophones représentent $63,4 \%$ des personnes. Le Pays Basque dans son ensemble est plus bascophone.

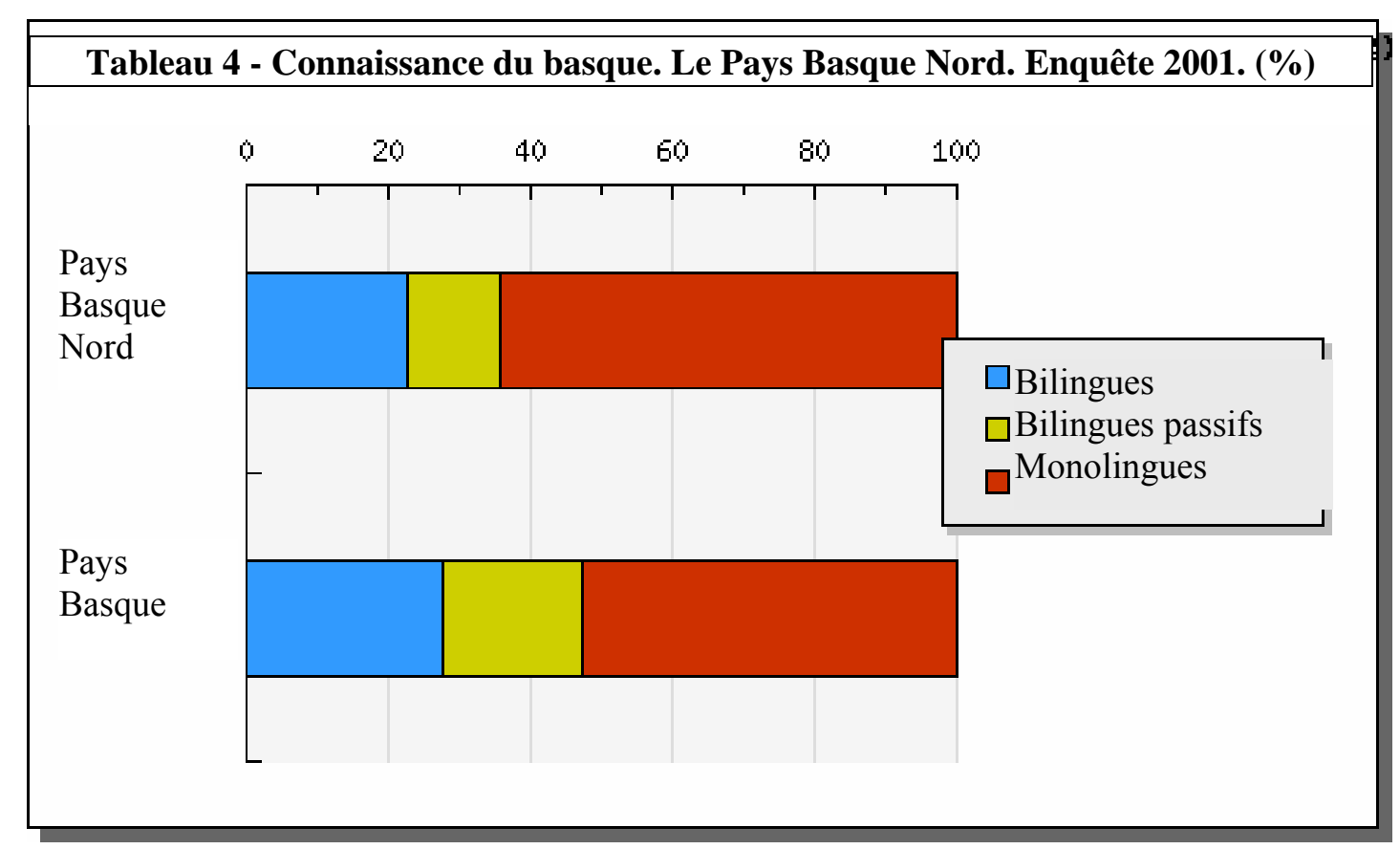

Le tableau 5 indique la proportion des trois types de locuteurs selon l'âge en Pays Basque Nord. On observe que plus on descend en âge et moins on connaît la langue. 


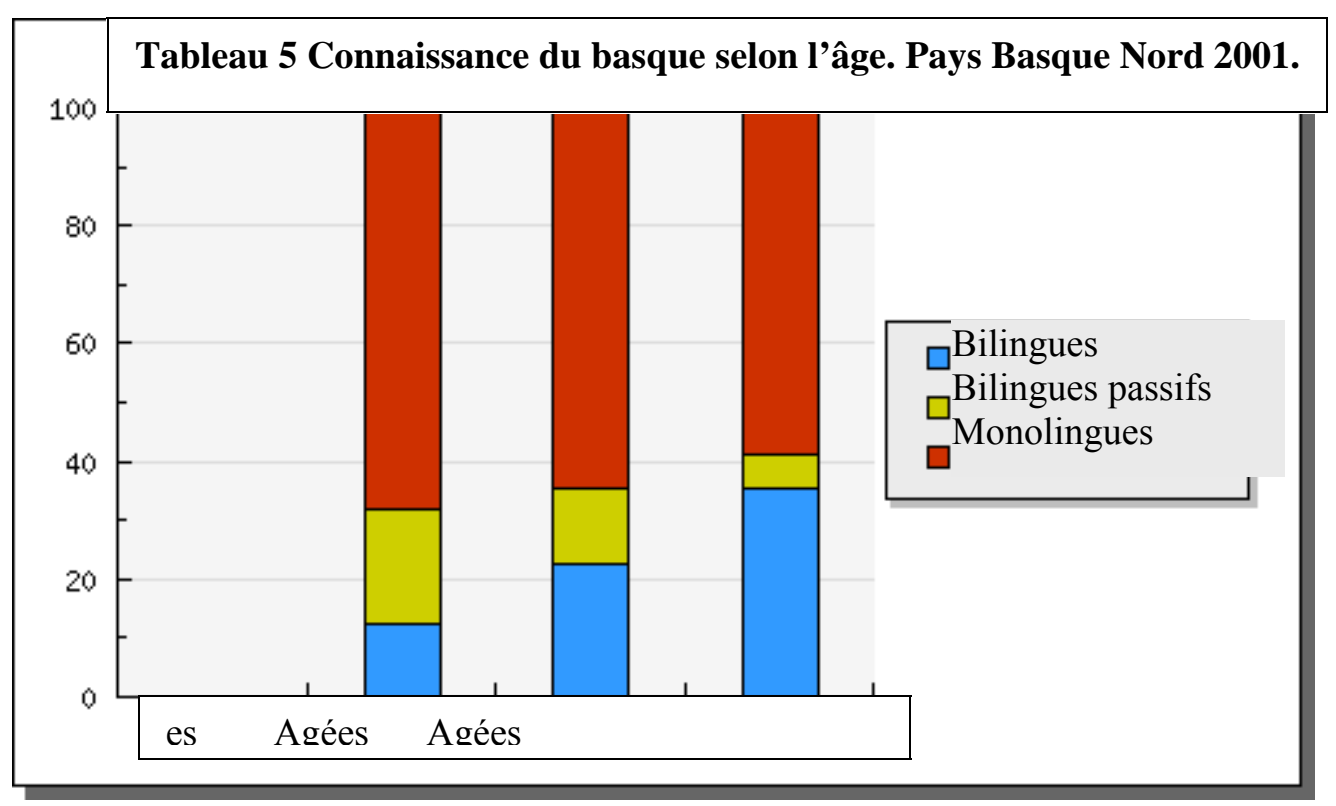

En Guipúzcoa, par exemple, nous n'en donnons pas le tableau, c'est l'inverse et ceci est directement lié au développement de l'enseignement scolaire de la langue. Nous retiendrons que la situation est inversée entre le Pays Basque Nord et la Communauté d'Euskadi. Dans le premier, ce sont surtout les personnes âgées qui connaissent le basque, acquis oralement en famille, l'enseignement n'étant pas assez développé pour compenser les pertes. En Euskadi, c'est l'inverse, ce sont surtout les jeunes qui connaissent le basque, appris à l'école, alors que le basque s'était plus perdu parmi les personnes âgées.

Notons que selon 1'Atlas des langues en péril dans le monde publié par l'Unesco en $2001^{6}$, à part le corse, tous les langues minorisées de l'hexagone risquent de disparaître. Un des critères retenus par l'Unesco pour décider qu'une langue est en danger est que moins de $30 \%$ des jeunes ne la parlent. C'est le cas du basque en France.

Selon l'enquête intitulée «Etude de l'histoire familiale de 1999 », réalisée avec le recensement de 1999, qui comportait un volet consacré à la «transmission familiale des langues et parlers » conçue avec l'Institut d'études démographiques et réalisée par l'Insee, « la probabilité d'avoir été élevé dans une langue régionale est multipliée par dix pour les natifs de Corse ou d'Alsace. Elle est également plus élevée qu'ailleurs pour les natifs des Pyrénées-Atlantiques », donc pour le basque ${ }^{7}$.

De même l'étude intitulée «La dynamique des langues en France au fil du XXe siècle ${ }^{8}$ indique page 3 que la transmission familiale de l'alsacien a lieu " plus souvent sur un mode habituel (660 000 personnes) qu'occasionnel (240 000), c'était encore il y a une génération la langue régionale la mieux transmise. (...) Dans le reste de la France, seul le basque a connu une situation analogue ". Il serait intéressant de savoir si, à l'heure actuelle, cette transmission diminue ou pas, or on manque d'étude ciblée sur ce point.

\section{2. Évaluation de l'usage de la langue basque}

Nous allons maintenant nous intéresser brièvement au deuxième point, c'est-à-dire à l'usage qui est fait de la langue basque. Cet aspect est encore plus difficile à mesurer que la connaissance de la langue. L'Institut de sociolinguistique basque mène depuis 1989 des

\footnotetext{
${ }^{6}$ 1ère édition en 1996.

${ }^{7}$ Langues régionales, langues étrangères : de l'héritage à la pratique, INSEE Première, p. 3.

${ }^{8}$ Heran et al.
} 
enquêtes in situ, dans la rue, tous les quatre ans. Des observateurs notent en direct l'usage spontané de la langue des personnes qu'ils repèrent.
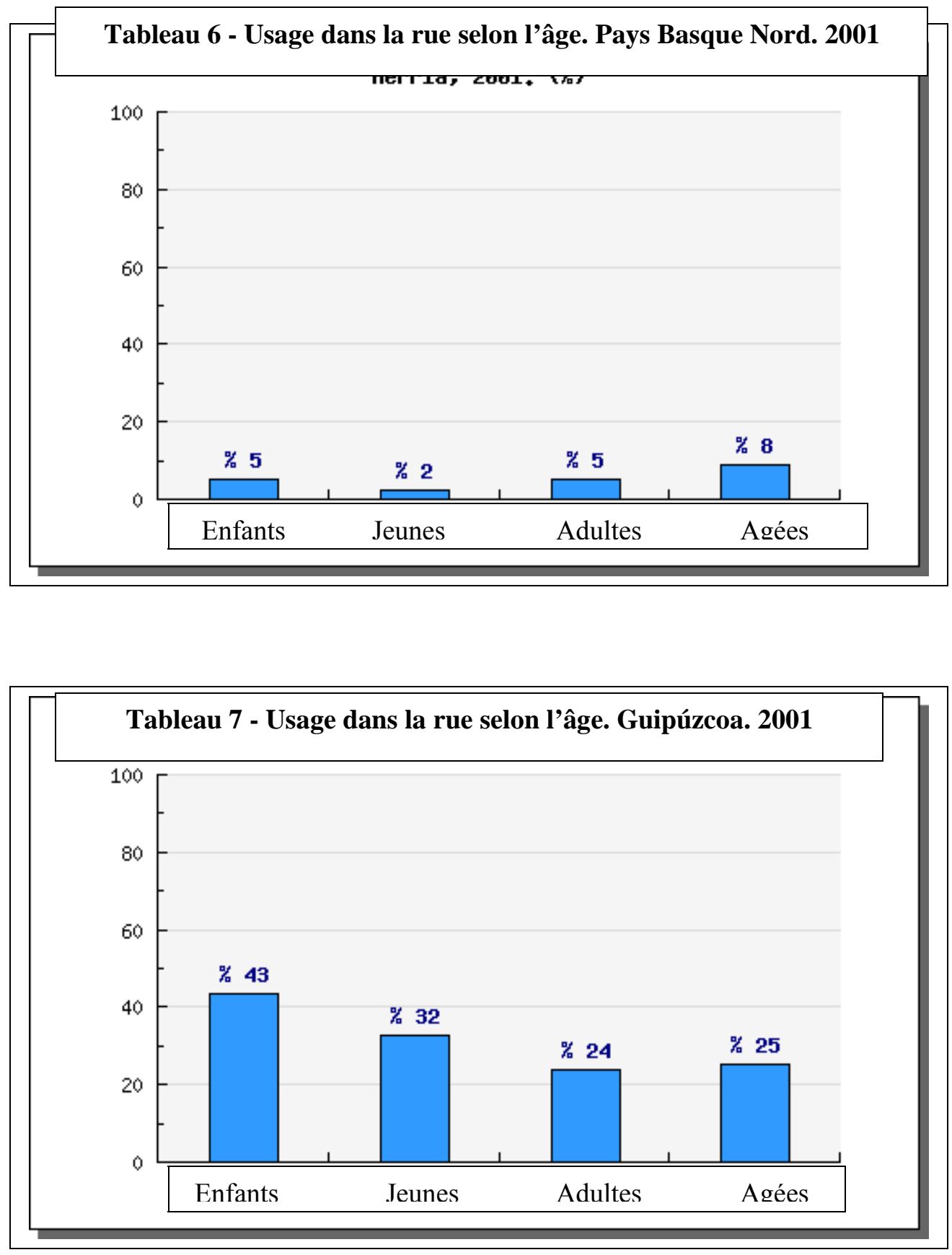

Les deux derniers tableaux ci-dessus, tableaux 6 et 7, mettent à nouveau en évidence la grande différence entre le Pays Basque Nord et le Guipúzcoa. Dans le premier, la proportion de conversation en basque est faible, surtout chez les jeunes avec une légère remontée chez les enfants qui est sûrement un effet direct de la scolarisation en basque qui s'est développée. En Guipúzcoa, la proportion est beaucoup plus élevée chez les enfants, plus que chez les jeunes ou les personnes âgées.

On peut donc conclure qu'il y a un effet direct de l'enseignement de la langue sur son usage dans la rue, puisque la transmission familiale est peu développée, même en Guipúzcoa. 
Bien sûr bien d'autres types d'enquêtes et observations in situ devraient être menées pour évaluer les pratiques effectives dans les différents domaines de la vie privée et publique.

Les jeunes bascophones sont donc de plus en nombreux en Euskadi, mais dans les zones non-bascophones l'usage par les jeunes est anecdotique, et le risque est évident que le basque soit une simple matière scolaire et finalement que la motivation d'utiliser le basque se perde. D'après la grande enquête sociolinguistique de 2001, deux tiers des bilingues entre 2545 ans utilisent le basque à la maison, moins de la moitié des bilingues de moins de 25 ans utilise le basque à la maison.

Parmi les nouveaux bilingues tous âges confondus en Euskadi, soit 10,8\% de la population, on aurait $84,1 \%$ de bilingues passifs qui sont ceux qui comprenant la langue ne l'utilisent pas, et seulement $4 \%$ qui véritablement seraient bascophones actifs. Seulement $2 \%$ des nouveaux bascophones utilisent le basque à la maison et $17 \%$ l'utilisent avec le castillan, car leur famille est en général uniquement castillanophone9.

Voici quelques autres exemples. Il existe une chaîne de télévision financée par la Communauté autonome basque entièrement en basque depuis plus de 20 ans, ETB 1. Son audience globale qui était de 5,5\% en Euskadi en 1993 est de 5,3\% en 2004, malgré un pourcentage de bascophones en augmentation. C'est le samedi que l'audience est la plus forte avec les retransmissions sportives. Le même film d'Harry Potter doublé en basque et doublé en castillan a été diffusé le même jour par Euskal Telebista. On a évalué à 15000 les spectateurs pour la version basque et 302000 pour celle en castillan.

Parmi les habitants d'Euskadi qui utilisent Internet, 12,4 \% emploient le basque, 24\% l'anglais et 95\% le castillan. Parmi les 6-14 ans, 23,5\% utilisent le basque. On a donc ici une évolution positive ${ }^{10}$.

Selon une enquête concernant la lecture en Euskadi, en 2003, 32,6\% des habitants lisent en basque, $0,6 \%$ d'entre eux seulement en basque et $62,6 \%$ seulement en castillan. Parmi ceux qui lisent en basque, $48,1 \%$ le font pour améliorer leur niveau.

Ces différents exemples tendent à montrer que malgré une offre en basque qui augmente, des possibilités d'utiliser la langue dans des domaines de la vie privée et publique de plus en plus divers, l'usage ne décolle pas. Bien sûr ceci serait à étudier de plus prêt en diversifiant les domaines et les types d'études, avec une attention particulière sur les comportements linguistiques des jeunes.

\section{Aperçu des facteurs induisant l'écart entre connaissance et usage de la langue ${ }^{11}$}

$\mathrm{Au}$ vu des tableaux 5, 6 et 7, en tout cas, on constate un écart important entre la connaissance de la langue et son usage, ici dans la rue. Pour l'ensemble du Pays Basque, on évalue grossièrement la connaissance à $25 \%$ et l'usage quotidien à $14 \%{ }^{12}$. Ceci peut

\footnotetext{
${ }^{9}$ Mensuel Berripapera $\mathrm{n}^{\circ}$ 160, septembre 2005, Vice-Ministère de la Politique Linguistique, Gouvernement autonome basque.

${ }^{10}$ D'après Gazteen \%23k euskara erabiltzen du amaraunean, 06/09/2005, www.erabili.com/zer_berri/berriak/

${ }^{11}$ Nous avons fait depuis une communication plus technique sur cette thématique lors du colloque intitulé, Politique linguistique et enseignement des Langues de France, colloque organisé par l'Association Universitaire des Langues de France en mai 2005. Le point de départ choisi est le locuteur, que l'on peut « partager » en émetteur de messages linguistiques et récepteur. Les facteurs directs et indirects sont disposés en cercles concentriques et positionnés plus ou moins prêts de ce centre selon qu'ils agissent directement ou pas, en trois cercles : facteurs individuels propres au locuteur potentiel (compétence, attitude, perception) au centre, facteurs contextuels (d'énonciation) plus éloignés (en particulier ceux propres à l'interlocuteur) et facteurs socio-juridico-politico-économiques indirects à l'extérieur.

${ }^{12}$ Sareko Argia, 2002.
} 
s'expliquer au moins partiellement par un phénomène mécanique : étant donné que le nombre de personnes qui parlent le basque est inférieur à celui de celles qui parlent castillan ou français, on aura en théorie plus d'occasions de parler castillan ou français que basque. Plus précisément, c'est la densité de bascophones dans l'environnement du locuteur qui est un facteur induisant l'usage de la langue.

On observe aussi que les jeunes nouveaux bascophones parlent plus aisément en espagnol ou en français qu'en basque car leur compétence en basque est moins importante, le basque est une deuxième langue pour eux. Ceux issus de l'enseignement de type immersif sont en principe plus compétents que ceux issus du modèle bilingue ou d'initiation ou optionnel, mais parmi eux on trouve des niveaux très différents. Ainsi le Ministère de l'Education du Gouvernement autonome basque a fait passer en 2004 pour la première fois un examen établi selon des normes européennes aux élèves de 16 ans pour évaluer leur niveau : aucun élève étudiant selon le modèle A (basque comme simple matière) ne l'a réussi, $67 \%$ des élèves étudiant selon le modèle $\mathrm{B}$ (bilingue à parité) ont échoué et même $32 \%$ de ceux étudiant selon le modèle D (immersion) ${ }^{13}$.

Le milieu familial et plus généralement l'environnement linguistique extra-scolaire ont aussi une grande importance évidemment; si la langue est aussi la langue de la vie familiale, l'aisance est beaucoup plus grande. D'autres facteurs jouent comme les capacités scolaires personnelles, les conditions offertes par l'établissement scolaire lui-même.

D'une façon générale la compétence passive en basque, l'écoute et la lecture, est plus grande que la compétence active, l'écriture et surtout la pratique orale. De plus, si au plan des registres formels de la langue, il y a eu des avancées depuis que cette politique volontariste s'est mise en place, dans l'administration, dans l'enseignement, dans les médias, il y a eu recul dans la qualité, la diversité de la langue dans les registres informels, quotidiens, intimes, selon les spécialistes. Les interférences avec le castillan ou français sont plus nombreuses. C'est ce que l'on pourrait appeler le problème de la qualité, qui n'est pas seulement celui de la correction grammaticale et des registres de langue.

Ce déséquilibre dans le bilinguisme n'est pas le seul facteur, mais il a pour conséquence que les jeunes utilisent plus souvent l'espagnol ou le français que le basque, même entre eux alors qu'ils savent que l'interlocuteur connaît la langue basque. L'usage n'est donc pas naturel, il relève de la volonté, d'un choix militant. Les résultats sur le plan de l'usage, de la pratique ne sont pas à la hauteur des efforts fournis depuis bientôt trente ans ${ }^{14}$.

Il est évident que sans le développement important de l'enseignement, il n'y aurait pas à l'heure actuelle autant de bascophones actifs dans leur langue dans les divers domaines de la vie publique, même en Pays Basque Nord. Connaître le basque est donc un plus mais le castillan ou le français sont eux indispensables. En dehors de l'école, la connaissance de la langue basque n'est pas une condition indispensable à l'obtention de la plupart des emplois, ni à l'accès à la connaissance, aux technologies et aux loisirs en général. La dimension utilitaire de la langue est essentielle : pourquoi apprendras-je le basque si cela ne me sert pas pour obtenir un emploi, si ce n'est pas un plus dans ma future vie professionnelle ?

Sorti de l'école, le jeune a peu de possibilités de vivre en basque ses loisirs. Comme le dit de façon un peu moqueuse le sociolinguiste Mikel Zalbide, « en envoyant le lundi matin nos enfants à l'ikastola apprendre à dire dicotylédon en basque, pensons-nous que nous provoquons un changement dans l'environnement socioculturel à la maison, dans le voisinage, le cercle d'amis, le village ? $\gg{ }^{15}$.

\footnotetext{
${ }^{13}$ Voir Ikasleen euskara maila neurtu dute, 27/04/2005, www.erabili.com/zer_berri/berriak/. Examen établi sur la base du First Certificate in English, d'un niveau moins élevé que Euskararen Gaitasun Agiria (EGA). Ont été évalués la lecture, l'écriture, la compréhension, l'expression et la connaissance de la langue.

${ }^{14}$ Zalbide, 2003.

${ }^{15}$ Zalbide, 2004.
} 
La dimension identitaire a son importance aussi, parler basque c'est faire partie de la famille basque, il y a une sorte de reconnaissance sociale. Cette dimension identitaire est plus mise en avant selon les enquêtes par les jeunes en Pays Basque Nord qu'au Sud où c'est la dimension utilitaire de la langue qui est plutôt retenue par ceux-ci.

Pour conclure sur ce point, il faut donc souligner l'écart entre la connaissance et l'usage de la langue. Au vu des résultats des différentes enquêtes sociolinguistiques, on peut avec la linguiste Karmele Rotaetxe observer que « les données montrent une augmentation du nombre de bascophones d'intérêt puisqu'elle concerne les tranches d'âge des jeunes. Mais l'usage ne semble pas s'accroître au même rythme que la connaissance " (Résumé de l'article). Dit autrement connaissance ne signifie pas usage. L'utilisation scolaire de la langue est particulière puisqu'elle a pour objet d'apprendre la langue et non de s'en servir comme outil de communication, sauf dans le modèle immersif. Il s'agit d'améliorer sa compétence dans la langue pour peut-être l'utiliser ensuite. Il n'y a pas implication nécessaire de l'usage, même si la connaissance est indispensable à celle-ci ${ }^{16}$. Pour qu'il y ait usage, il faut des conditions socio-psychologiques favorables. En effet utiliser une langue relève et du choix individuel et du contexte socio-juridico-politico-économique...

Dans la Communauté autonome d'Euskadi également, « l'usage est encore faible et on peut se demander si l'insistance politique sur le côté symbolique du basque n'aurait-elle pas sous-estimé le besoin de l'utilisation de la langue, instrument primordial de communication $»^{17}$. «Plus que la dimension symbolique, c'est l'insistance sur les aspects formels en Euskadi, politiques, juridiques et législatifs, administratifs, qui ressort. Son effet d'entraînement, même si la reconnaissance par les pouvoirs publics était un point important vis-à-vis de la société civile, n'a pas atteint le niveau escompté, (...) il faut dire que la langue basque partait de loin par rapport au castillan $»^{18}$.

En France, par contre, même sur le plan formel, les avancées sont très timides et les blocages sont importants, en particulier au niveau de l'Etat en dehors de l'enseignement de la langue.

\section{Conclusion : Au-delà de l'enseignement de la langue, une politique linguistique globale}

En plus du développement de l'enseignement de la langue qui en fait partie, c'est donc une politique globale, adaptée à la situation linguistique qu'il faut mettre en œuvre. En Alsace du fait du voisinage avec l'Allemagne, elle ne sera pas la même que celle pour le basque qui ne peut compter que sur lui-même, la politique mise en œuvre au Euskadi bénéficiant directement ou indirectement à la langue basque en Pays Basque Nord. Pantxika Maitia, Responsable du Service de la langue basque à l'Institut culturel basque peut ainsi dire: " En tant qu'institution, nous avons une mission d'explication, nous devons faire comprendre que l'enseignement ne suffit pas, qu'il faut créer un environnement bascophone pour nos enfants. A nous de provoquer la prise de conscience, de l'accompagner, et d'être une force de proposition $»^{19}$.

Il faut mettre en place les conditions pour que la langue apprise soit utilisée, donc utile pratiquement ou symboliquement, qu'elle soit un outil de communication, d'expression. Nous ne pouvons pas exposer ici plus longuement quelles sont ces conditions.

\footnotetext{
${ }^{16}$ Comme le dit Iñaki Martinez de Luna dans SAREKo ARGIA, 2002, Euskararen erabilera, nous traduisons : «Parmi nous beaucoup sont capables de parler plus ou moins en anglais, mais cela ne nous vient pas à l'idée de le faire entre nous », p.2.

${ }^{17}$ Rotaetxe : 8 .

${ }^{18}$ Coyos, $2004: 102$.

${ }^{19}$ Leihotik, $\mathrm{n}^{\circ}$ 33, mai 2004, Institut Culturel Basque.
} 
On retiendra qu' " un levier clé, sinon le levier primordial de l'inversion du glissement linguistique, pour reprendre le concept de Fishman (...), c'est la transmission familiale, la transmission entre amis, dans les cercles proches de la vie privée. Ce que certains appellent la transmission naturelle quand on ne la cantonne pas à la transmission familiale $»^{20}$. L'apprentissage de la langue en milieu familial, surtout si les deux parents parlent la langue, est bien plus efficace que l'apprentissage en milieu scolaire. Je cite le psycholinguiste Jean Petit récemment décédé : «Un milieu familial normal offre les conditions idéales au déploiement de la stratégie naturelle de l'acquisition. Un apprenant unique est en effet en contact avec au moins deux personnes lui servant de modèle linguistique. Dans une classe immersive, la situation est toute différente. Le seul modèle présent est l'enseignant qui trouve en face de lui une vingtaine d'apprenants ou davantage $\gg{ }^{21}$.

Mais ce sera la conclusion, l'enseignement s'avère indispensable quelle que soit la situation de la langue considérée dans le monde moderne. Il l'est encore plus dans le cas des langues peu ou pas transmises en famille. Ce sera un enseignement adapté tenant compte du fait que les élèves ne sont pas ou peu en contact avec la langue en dehors de l'école.

\section{Références bibliographiques}

Coyos Jean-Baptiste, 2004, Politique linguistique Langue basque et langue occitane du Béarn et de Gascogne, Saint-Sébastien / Bayonne, Editions Elkar, 260 p.

, à paraître, Ecart entre connaissance et usage d'une langue minoritaire : essai de typologie des facteurs. Le cas de la langue basque, Politique linguistique et enseignement des Langues de France, Colloque organisé par l'Association Universitaire des Langues de France, Toulouse (France), 26-27/05/2005.

Euskal Soziolinguistika Institutua SEI [Institut de sociolinguistique basque], 2002, Kale erabileraren IV. neurketa 2001. Emaitzak, Azterketak, Gogoetak [4 $4^{\text {ème }}$ mesure de l'usage de la langue basque dans la rue 2001. Données, Etudes, Réflexions], Bat Soziolinguistika aldizkaria, 43, Euskal Soziolinguistika Institutua, Andoain, 151 p. (site en basque Web de l'institut: www.sortu.org).

, Euskararen Datu-Basea [Base de données sur la langue basque], Graphiques, schémas sur l'enseignement et l'usage de la langue selon divers paramètres, en basque, www.sortu.org/edb/.

Fishman Joshua A., 1991, Reversing Language Shift. Theoretical and Empirical Foundations of Assistance to Threatened Languages, Clevedon/England, Multilingual Matters, $431 \mathrm{p}$.

, 2001, Can Threatened Languages be saved ? Reversing Language Shift revisited: A $21^{\text {st }}$ Century Perspective, Clevedon/England, Multilingual Matters, $492 \mathrm{p}$.

Garzia J., Egaña A. et Amonarriz K., 2004, Euskararen kalitatea : zertaz ari garen, zergatik eta zertarako [La qualité de la langue basque : de quoi s'agit-il, pourquoi et dans quel but ?], 29/10/2004, www.erabili.com.

Gouvernement BASQue, 2003, Euskararen Jarraipena III - La Continuité de la Langue Basque III, Euskal Herriko Soziolinguistikako Inkesta 2001 - Enquête Sociolinguistique au Pays basque 2001, quatre volumes (quadrilingue), chacun 56 p.+CD-Rom (ou sur www.euskadi.net/euskara).

Heran François, Filhon Alexandra, Deprez Christine, 2002, La dynamique des langues en France au fil du XXe siècle, Populations \& Sociétés, $n^{\circ} 376$, Institut national d'études démographiques, $4 \mathrm{p}$.

\footnotetext{
${ }^{20}$ Coyos, $2004: 199$.

${ }^{21}$ Petit : 30 .
} 
INSTITUT NATIONAL DE LA STATISTIQUE ET DES ÉTUDES ÉCONOMIQUES, 2002 février, Langues régionales, langues étrangères : de l'héritage à la pratique, INSEE Première, ${ }^{\circ} 830,4$ p.

Office Public de la langue basque / Euskararen erakunde Publikoa, 2005, Diagnostic de l'offre d'enseignement du basque et en basqueAnnée scolaire 2004-2005 / Euskararen eta euskarazko irakaskuntza eskaintzaren azterketa kuatitatiboa 2004-2005 ikasturtea, polycopié, bilingue, $80 \mathrm{p}$.

, 2005, L'offre d'enseignement du basque et en basque Volet 1 de la programmation pluriannuelle / Euskararen eta euskarazko irakaskuntzaren eskaintza Epe luzeko programazioa 1. zatia, polycopié, bilingue, $336 \mathrm{p}$.

Peтіт Jean, 2001, L'immersion, une révolution, Colmar, Jérôme Do. Bentziger éditeur, 198 p.

RotaetXe Karmele, 1997, Connaissance et usage d'une langue minoritaire : le cas basque, Proceedings of the 16th International Congress of Linguists - Paris, Oxford, Pergamon, Paper $\mathrm{n}^{\circ} 0485,11 \mathrm{p}$.

Sareko Argia, Aizpurua Xabier, Altuna Olatz, Martinez de Luna Iñaki, Ramos Rosa, 2002, Euskararen erabilera [L'usage de la langue basque], Mahaikideak Mahaingurua Iritziak [Participants Table ronde Opinions], Larrun, 56, 30/06/2002, 14 p., www.argia.com/larrun/htdocs/euskaerabili2.htm.

United Nations Educational Scientific and Cultural Organization, 2001 (1996), Atlas des langues en péril dans le Monde, par Stephen A. Wurm, 60 p.

ZaLbIDE Mikel, 2003, Ahuldutako Hizkuntza Indarberritzea: teoriak zer dio? [Le renforcement des langues menacées: que dit la théorie ?], 07/07/2003, www.erabili.com.

,2004, Joshua A. Fishman-en RLS edo HINBE (I, II, III, IV, V, VI) [Le RLS ou

HINBE de Joshua A. Fishman], 07/04/2004 à 08/06/2004, www.erabili.com. 